\title{
Influence of electrode material in micro-EDM drilling of stainless steel and tungsten
}

\section{carbide}

G. D’Urso' ${ }^{1}$, G. Maccarini ${ }^{2}$ C. Ravasio ${ }^{3}$

${ }^{1}$ University of Bergamo - DIGIP - Via Pasubio 7b, 24044 Dalmine (BG) - Italy durso@unibg.it

${ }^{2}$ University of Bergamo - DIGIP - Via Pasubio 7b, 24044 Dalmine (BG) - Italy giancarlo.maccarini@unibg.it

${ }^{3}$ University of Bergamo - DIGIP - Via Pasubio 7b, 24044 Dalmine (BG) - Italy chiara.ravasio@unibg.it

Keywords: micro-EDM, micro-Drilling, Tungsten Carbide, Stainless Steel, Process Performance, Electrode

Abstract

The aim of this work is to analyse the influence of electrode material in micro-EDM drilling of stainless steel and tungsten carbide metal plates. Brass and tungsten carbide electrodes were selected basing on their different electrical and thermal properties. The experimental campaign was based on the execution of a large number of micro-holes carried out by varying some process parameters, namely peak current, voltage and frequency. Tool wear ratio and material removal rate were assumed as process performance indexes, while diametral overcut and taper rate were considered as geometric indexes. The Analysis of Variance (ANOVA) was applied on the results coming out from the experiments. Moreover, a overall Process Index based on both process parameters and electrode material properties 
was proposed. This index fits, with a good correlation level, all the process performance and geometric indicators for both the considered workpiece materials. A comparison between the performance measured using stainless steel and tungsten carbide workpieces was also carried out. The results of this experimental research showed that electrical resistivity, thermal conductivity and melting point of both electrode and workpiece materials have a significant effect on the process and geometric performance.

\section{Introduction}

The trends for miniaturization of components, machine parts and devices continuously forces the search and development of new manufacturing solutions or the improvement of existing methods for their production, as declared by Trych (2013). Several researches in this field, like for example the state of the art of micro production technology made by Weule et al. (2004), confirmed that the need for products containing micro-features has shown a noticeable and continuous growth in many fields of application and, within the different types of micro-feature, the need for products containing micro-holes has shown remarkable growth in some industrial sectors. Micro holes are widely used for the production of several industrial components such as medical and optical devices, turbine blades, cooling channels, diesel fuel injection nozzles; as an example, the problems related to the drilling execution in fuel injection nozzles were studied by Tong and $\mathrm{Li}$ (2013).

At the same time, materials with distinctive mechanical and physical properties, such as titanium or nickel based alloys, tungsten carbide and its composites, tool steels and other super alloys, have been developed to meet the needs of specific applications. In general, as demonstrated through the studies performed by Jahan et al. (2011) on tungsten carbide and by Schuberta (2013) on zirconia ceramics, these materials have high performing properties in terms of hardness, toughness, low heat sensitivity, high fatigue and corrosion resistance 
with respect to other more common materials, even though they are often more difficult to be machined.

A large number of researches have been carried out to study machinability of this material using both conventional and non-conventional processes. Among conventional technologies, the experiments performed on grinding by Mahdavinejad (2005) and on turning by Liu and Li (2011) lead only to partial good results. Problems related to very low material removal rate, depth of cut and tool durability were observed also by Liu et al. (2004) and Engqvist et al. (1999). On the opposite, non-conventional technologies proved to be suitable not only to overcome the problems related to very hard materials but also for applications where dimensional accuracy and complex geometries are primary requests.

Among the non-conventional methods, micro electro-discharge machining (EDM) is one of the most performing methods capable of machining difficult to cut materials and to achieve, at the same time, the dimensional and geometrical performances usually requested by microscale components.

The EDM process is based on removal of material through melting and evaporation. The electrical discharges occur between electrode and workpiece in a dielectric medium that separates the two elements. A voltage is attached to both electrodes and, when the breakdown voltage of the medium is reached, a plasma channel allowing for a current flow is established and a discharge takes place. The material is melted and vaporized because of the high temperature generated by the electrical discharges; in these conditions the electrode material is also melted and vaporized, giving rise to a remarkable electrode wear. Micro EDM is considered one of the most significant technologies in micro-drilling, especially for very small and very high aspect ratio burr-free micro-holes. This technology can be applied on every conductive materials independently by the mechanical and physical properties. As affirmed by Sona et al. (2007), since micro EDM is a contact-less process, it is possible to 
fabricate micro parts and micro-scale features without distortion or brakeage due to physical forces. The tool wear, that can achieve very high values, and the machining time, that in some cases can even limit the application of this process to industrial needs are the two main critical aspects to consider when this technology is used.

The EDM process is essentially a thermal process and it is based through the control of several parameters such as voltage, current, pulse duration, sparking gap and frequency. As described in the studies conducted by Jahan et al. (2009) and Lee and Li (2003), these parameters have effects on the erosion process in terms of tool wear ratio and material removal rate; at the same time they can affect the properties of the machined workpiece in terms of surface finishing, dimensional and geometrical accuracy. For example, as described in D’Urso et al. (2014), during the execution of a deep hole, the debris accumulation at the bottom of the hole may cause abnormal discharges, resulting in machining speed reduction and in geometrical and dimensional alteration.

Concerning the performance of the micro EDM drilling, as confirmed by Jahan et al. (2009), suitable process parameters are essential to achieve high material removal rate (MRR) and limited tool wear ratios (TWR). Concerning this aspect, also Denkena et al. (2006) carried out many studies for the optimization of micro-drilling process by adequate experiments. Pradhan et al. (2009) performed an investigation on micro electro discharge drilling of a titanium super alloy and it was showed that machining performance is mostly affected by peak current and pulse on time. Using EDM technology with a pure electrolytic copper electrode, Kuppan et al. (2008) carried out small deep holes were on Inconel 718, by varying peak current, pulse on time, duty factor and electrode speed. The results demonstrated that MRR is mainly affected by peak current, duty factor and electrode rotation, while surface roughness is strongly influenced by peak current and pulse on time. Concerning the electrode, Jahan et al. (2009) demonstrated that both geometry and material have an 
influence on MRR, electrode wear and surface finishing. For example, Yilmaz and Okka (2010) observed that single-channel electrodes resulted to provide better material removal rates and lower electrode wear ratios than multi-channel tubular electrodes. However multichannel electrodes produce better surfaces and lower hardness values than single-channel electrodes. With regards to the electrode material, the experiments described by D'Urso et al. (2011) demonstrate that, for titanium alloy workpieces, brass and copper electrodes provide minimum machining time while machining time increases a lot using special carbide electrodes; on the contrary, minimization of tool wear ratio can be obtained using special carbide electrodes with respect to copper and brass. Finally, it must be considered that workpiece material properties can also influence the final output.

The dimensional and geometrical aspects of the micro-holes are often described by two different indicators. The first one is the Diametrical Overcut (DOC) which gives information about the difference between the effective top diameter and the nominal electrode diameter. The overcut is influenced by the sparking gap due to the process parameters, it affects the hole dimensional accuracy and becomes important when high precision components are concerned. Several studies conducted by Yonga et al. (2013) have demonstrated that the overcut is associated with many variables, such as discharge voltage, current, duty cycle, and pulse duration. The second geometrical indicator is the Taper Rate (TR), which gives information about the tapering of the holes. The holes usually have the top diameter larger than the bottom one as for example described by Hwang et al. (2010), but in other cases, as reported by Chiang and Wang (2011), it was observed an inverse-taper of hole shape, due to the discharge between processing debris and the hole's lateral wall. Based on these considerations, it is possible to assert that the actual effects of micro-EDM process parameters, electrodes and workpiece materials on the final output are not fully known and have often yet to be clarified. Moreover, Hoffmeister and Illenseer (2004) 
affirmed that optimal process conditions for micro-drilling has often to be determined by the help of experiments, because of the unhelpfulness of the conventional drilling standard process parameters and the lack of suitable manufacturers parameter data.

The present paper aims to study the influence of electrode material properties in micro-EDM drilling of stainless steel and tungsten carbide workpieces. Brass and tungsten carbide electrodes, having different electrical and thermal properties, were tested. The experimental campaign was carried out by executing several micro-holes using different process parameters, namely peak current, voltage and frequency. Some process performance indicators, such as tool wear ratio and material removal rate, and some geometric indicators such as diametral overcut and taper rate were taken into account. The Analysis of Variance (ANOVA) was applied on the results coming out from the experiments and some correlations among process parameters workpiece materials and electrode materials were found. An overall Process Index based on both process parameters and electrode material properties was finally proposed.

\section{Experimental set up}

The micro EDM machine used in the experimental campaign was a Sarix SX-200. The micro holes were executed on both stainless steel (AISI 304) and tungsten carbide plates having a thickness equal to $3 \mathrm{~mm}$. Two electrodes' materials, brass and tungsten carbide, having different thermal, physical and electric characteristics, were used (Table 1). Both electrodes were tubular, with an outer diameter equal to $0.3 \mathrm{~mm}$ and an inner diameter equal to 0.12 $\mathrm{mm}$. The experimental campaign was carried out by varying some process parameters, namely peak current (I), voltage (V) and frequency (F). 
Hydrocarbon oil was used as dielectric and an internal electrode washing pressure equal to 6 bar was set when using stainless steel as workpiece, while 30 bar was set for tungsten carbide plates.

Table 1: Physical properties of electrodes' material.

\begin{tabular}{lcc}
\hline Physical Property & Brass C26800 & $\begin{array}{c}\text { Tungsten carbide } \\
\text { WC94Co6 }\end{array}$ \\
\hline Density $\left[\mathrm{g} / \mathrm{cm}^{3}\right]$ & 8.47 & 14.8 \\
Melting temperature $\left[{ }^{\circ} \mathrm{C}\right]$ & $905-930$ & 2867 \\
Electrical resistivity $[\Omega \mathrm{cm}]$ & $6.63 * 10^{-6}$ & $20 * 10^{-6}$ \\
Thermal conductivity $[\mathrm{W} / \mathrm{mK}]$ & 121 & 70 \\
Specific heat $\left[\mathrm{J} /\left(\mathrm{g}^{\circ} \mathrm{C}\right)\right]$ & & 0.3 \\
\hline
\end{tabular}

*Source: www.matweb.com

The process parameters kept fixed, as a function of both the electrode used and the workpiece, are reported in Table 2. Peak current, voltage and frequency were varied basing on a Design of Experiments (DOE) procedure with two levels, central point and five repetitions (Table 3 and 4). It must be noted that the fixed parameters and the ranges of the variable ones differ for the two electrode materials; this solution was used in order to test suitable technological windows for both materials. The execution order of the experiments was randomized to avoid possible systematical errors. The combination of the variable parameters resulted into 15 different technologies and in a total amount of 100 micro holes for each combination electrode-workpiece. 
Table 2: Fixed process parameters.

\begin{tabular}{|c|c|c|c|c|}
\hline Tool type & Bras & 800 & Tungsten & e WC94Co6 \\
\hline Workpiece & AISI304 & WC & AISI304 & WC \\
\hline Energy & 365 & 365 & 365 & 365 \\
\hline Polarity & - & - & - & - \\
\hline Width $[\mu \mathrm{s}]$ & 4 & 3.8 & 4.7 & 5 \\
\hline Gain & 120 & 120 & 50 & 40 \\
\hline Gap [\%] & 50 & 5 & 70 & 20 \\
\hline $\begin{array}{l}\text { Spindle rotational } \\
\text { speed }\end{array}$ & $100 \%$ & $100 \%$ & $100 \%$ & $100 \%$ \\
\hline Regulation & 03-01 & 03-01 & 03-01 & 03-01 \\
\hline
\end{tabular}

Table 3: Combination of Technologies based on DOE for AISI304 workpiece.

\begin{tabular}{l|ccc|ccc}
\hline Tool type & \multicolumn{3}{c|}{ Tungsten Carbide } & \multicolumn{3}{c}{ Brass } \\
& I [index] & V [V] & F [kHz] & I [index] & V [V] & F [kHz] \\
\hline Tech.1 & 40 & 110 & 110 & 65 & 110 & 130 \\
Tech.2 & 40 & 93 & 110 & 65 & 135 & 113 \\
Tech.3 & 40 & 127 & 110 & 65 & 135 & 130 \\
Tech.4 & 40 & 110 & 127 & 65 & 135 & 147
\end{tabular}




\begin{tabular}{l|lll|lll} 
Tech.5 & 40 & 110 & 93 & 65 & 160 & 130 \\
Tech.6 & 50 & 120 & 100 & 40 & 135 & 130 \\
Tech.7 & 50 & 100 & 120 & 50 & 120 & 140 \\
Tech.8 & 50 & 120 & 120 & 50 & 120 & 120 \\
Tech.9 & 50 & 100 & 100 & 50 & 150 & 140 \\
Tech.10 & 30 & 100 & 100 & 80 & 120 & 140 \\
Tech.11 & 30 & 120 & 120 & 80 & 150 & 140 \\
Tech.12 & 30 & 100 & 120 & 80 & 150 & 120 \\
Tech.13 & 30 & 120 & 100 & 90 & 135 & 130 \\
Tech.14 & 57 & 110 & 110 & 80 & 120 & 120 \\
Tech.15 & 23 & 110 & 110 & 50 & 150 & 120 \\
\hline
\end{tabular}

Table 4: Combination of technologies based on DOE for tungsten carbide workpiece.

\begin{tabular}{l|ccc|ccc}
\hline Tool type & \multicolumn{3}{c|}{ Tungsten Carbide } & \multicolumn{3}{c}{ Brass } \\
\hline & I [index] & V [V] & F [kHz] & I [index] & V [V] & F [kHz] \\
\hline Tech.1 & 40 & 110 & 83 & 26 & 120 & 130 \\
Tech.2 & 30 & 100 & 90 & 40 & 100 & 110 \\
Tech.3 & 50 & 100 & 90 & 40 & 140 & 110 \\
Tech.4 & 30 & 120 & 90 & 40 & 100 & 150 \\
Tech.5 & 50 & 120 & 90 & 40 & 140 & 150 \\
Tech.6 & 40 & 93 & 100 & 60 & 120 & 96 \\
Tech.7 & 23 & 110 & 100 & 60 & 86 & 130 \\
Tech.8 & 40 & 110 & 100 & 60 & 120 & 130 \\
Tech.9 & 57 & 110 & 100 & 60 & 154 & 130 \\
Tech.10 & 40 & 127 & 100 & 60 & 120 & 160 \\
Tech.11 & 30 & 100 & 110 & 80 & 100 & 110 \\
Tech.12 & 50 & 100 & 110 & 80 & 140 & 110 \\
Tech.13 & 30 & 120 & 110 & 80 & 100 & 150 \\
& & & & &
\end{tabular}




\begin{tabular}{l|lll|lll} 
Tech.14 & 50 & 120 & 110 & 80 & 140 & 150 \\
Tech.15 & 40 & 110 & 117 & 94 & 120 & 130 \\
\hline
\end{tabular}

A program for the automatic execution of the holes using the different technologies was implemented into the Sarix EDM machine. This program permits to record the machining time and the frontal electrode wear for each micro hole. At the end of each hole, the electrode clamp was moved to a specific reference point in order to measure the electrode wear by an electrode touching operation. The frontal tool wear (TW) was calculated as difference between the initial and the final length of the electrode. At the end of each hole drilling, the electrode tip was cut using the wire EDM unit to restore the same initial conditions for all the tests.

\section{Evaluation of the process performance and the geometrical characteristics of micro}

\section{holes}

MRR and TWR were assumed as performance criteria for the micro-holes drilling. Due to the debris movement and secondary discharge occurring between the hole and the electrode side, the machined hole is not cylindrical. In general, the diameter of the drilled hole is larger at the top, it decreases along the hole depth and it is minimum at the bottom. For this reason the diameter was measured at both the top and the bottom of each hole through an optical measuring microscope at the magnification of $100 \mathrm{X}$.

MRR was calculated as the ratio between the material removed from the workpiece $\left(\mathrm{MR}_{\text {workpiece, }}\right.$ estimated as the frustum of cone volume) and the machining time recorded by the EDM system (Eq. 1). 
$M R R=\frac{M R_{\text {workpiece }}}{t}=\frac{\pi h\left(D_{\text {top }}^{2}+D_{\text {top }} D_{\text {bottom }}+D_{\text {bottom }}^{2}\right)}{12 t}$

where $h$ is the thickness of the plate, $D_{\text {top }}$ and $D_{\text {bottom }}$ are respectively the top and bottom diameters of the hole and $t$ is the machining time recorded by the EDM system. TWR was calculated as ratio between the material removed from the electrode $\left(\mathrm{MR}_{\text {tool }}\right)$ and the material removed from the workpiece as given in Eq. 2.

$T W R=\frac{M R_{\text {tool }}}{M R_{\text {workpiece }}}$

The material removed from the tool is calculated as the volume of the tube having diameters equal to the nominal inner and outer electrode diameters (respectively $D_{\text {int }}$ and $D_{\text {ext }}$ ) and height equal to the measured tool wear $\left(h_{\mathrm{t}}\right)$, as reported in Eq. 3.

$M R_{\text {tool }}=\frac{\pi h_{t}\left(D_{\text {ext }}^{2}-D_{\mathrm{int}}^{2}\right)}{4}$

As regards the geometrical characteristics of micro-holes, Diametral Overcut (DOC) and Taper Rate (TR) were taken into account and calculated using Eq. 4-5.

$$
\begin{gathered}
D O C=100 * \frac{D_{t o p}-D_{e x t}}{D_{e x t}} \\
T R=\frac{D_{t o p}-D_{b o t t o m}}{h}
\end{gathered}
$$




\section{Analysis of the results}

The Analysis of Variance (ANOVA) was applied on the results coming out from the experiments. Considering that the analysis of the results focused at pointing out the effects of the power discharge and the electrode material properties on both the process performance and the geometrical characteristics of the micro holes, in order to represent the experimental conditions for each workpiece material, an overall index named "Process Index" was proposed. This index, taking into account the process parameters and the electrode material used, is defined as follows:

$$
I_{\text {process }}=\frac{I * V * F}{\rho_{e l} * k_{\text {ter }}}
$$

where $\rho_{\mathrm{el}}$ is the electrical resistivity and $\mathrm{k}_{\mathrm{ter}}$ is the thermal conductivity of the electrode. A similar approach was suggested by Mahardika et al. (2008), where an index based on the physical properties of the workpiece material was defined.

\subsection{Process performance evaluation for stainless steel workpiece}

Figures 1-4 show the interaction plots for MRR and TWR obtained on stainless steel using tungsten carbide and brass electrodes respectively. All the data refers to the average of five repetitions for each tested condition As confirmed by ANOVA, the considered process parameters significantly affect MRR and TWR for both the electrode types. It must be pointed out that the use of brass electrode causes a large data scatter in machining time and therefore in MRR. 


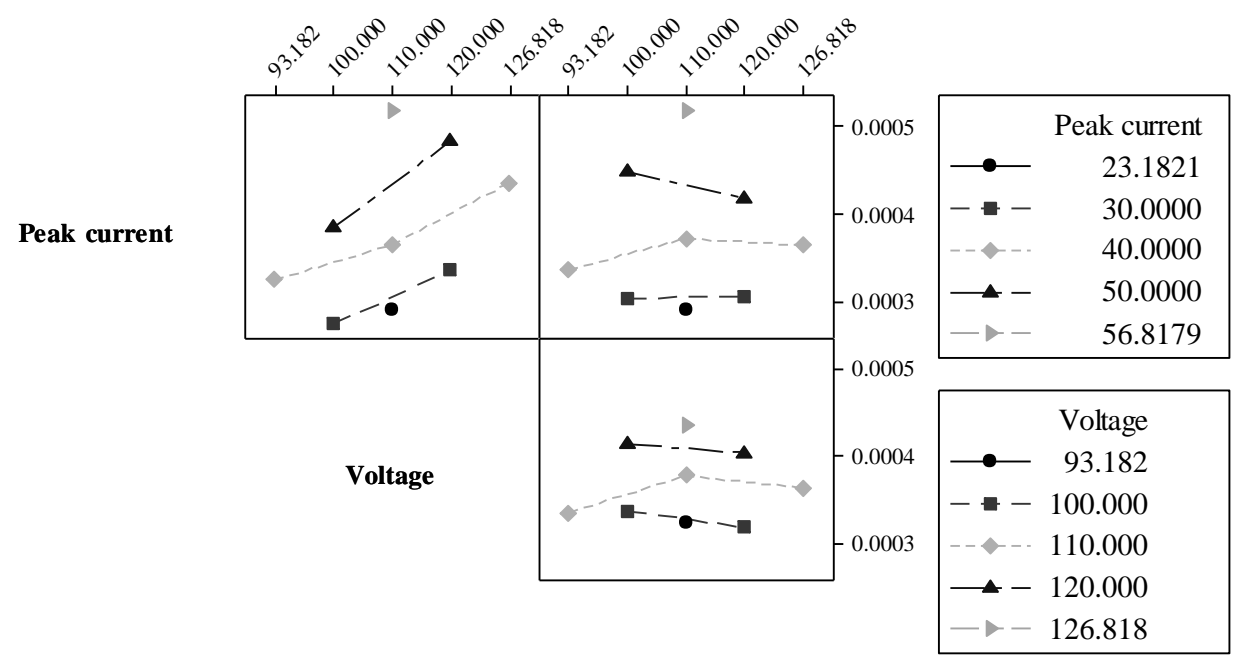

Frequency

Figure 1: MRR interaction plots for stainless steel using tungsten carbide electrode.

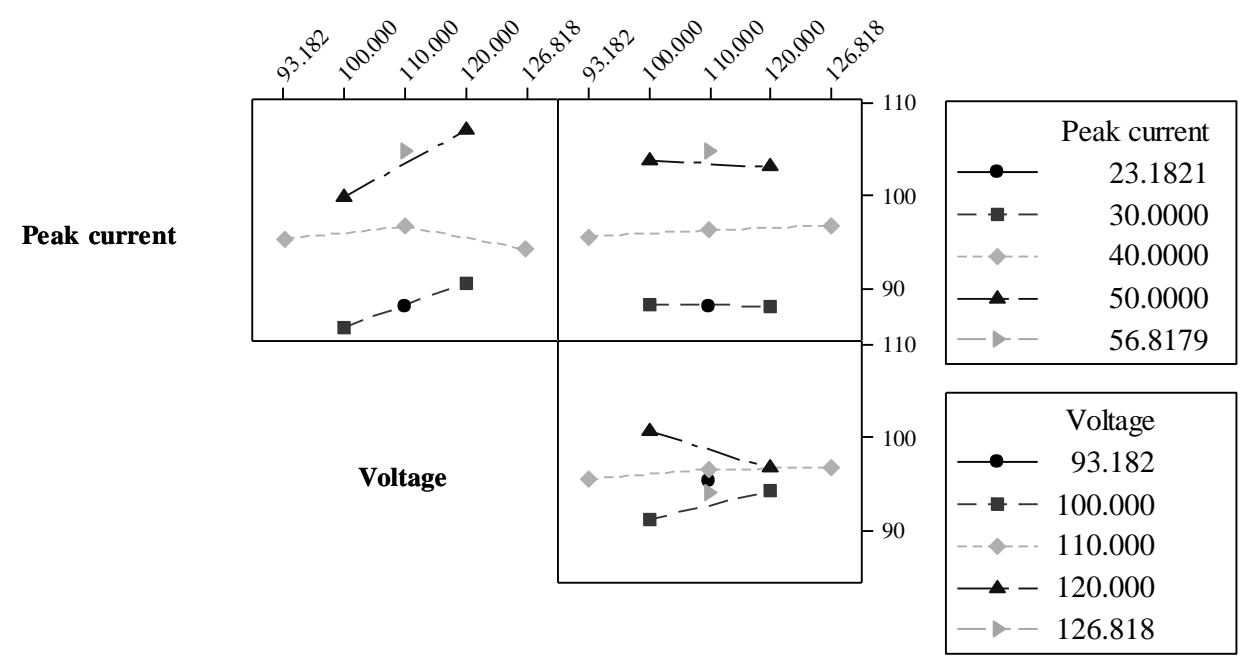

Frequency

Figure 2: TWR interaction plots for stainless steel using tungsten carbide electrode. 


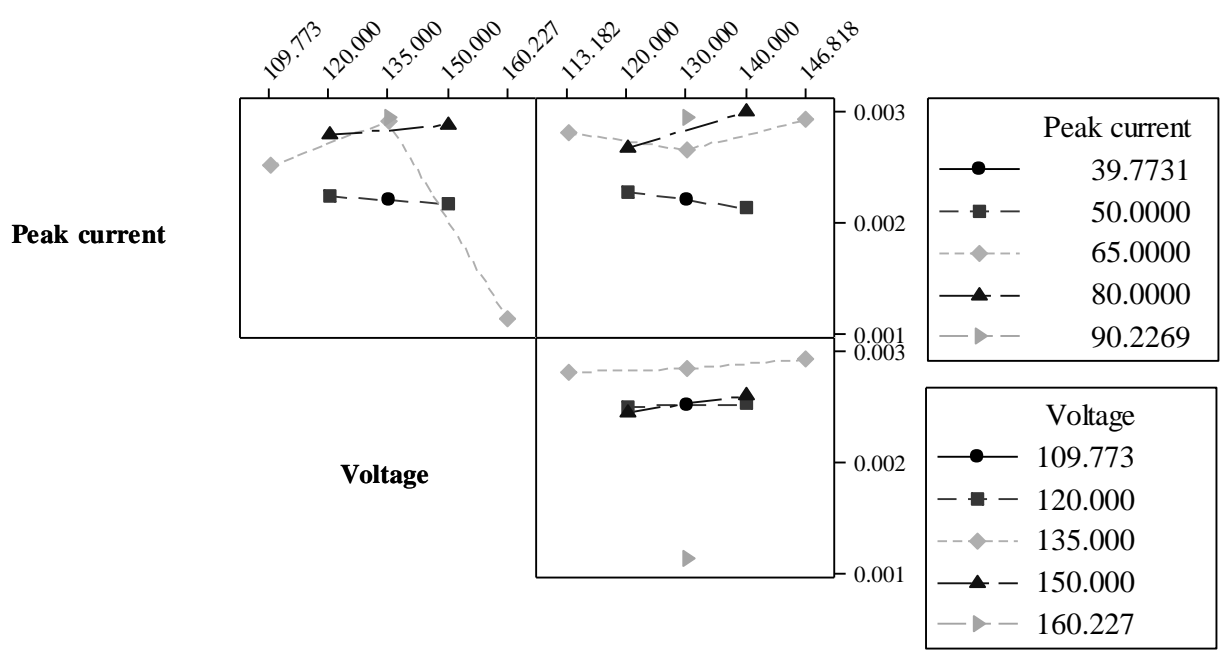

Frequency

Figure 3: MRR interaction plots for stainless steel using brass electrode.

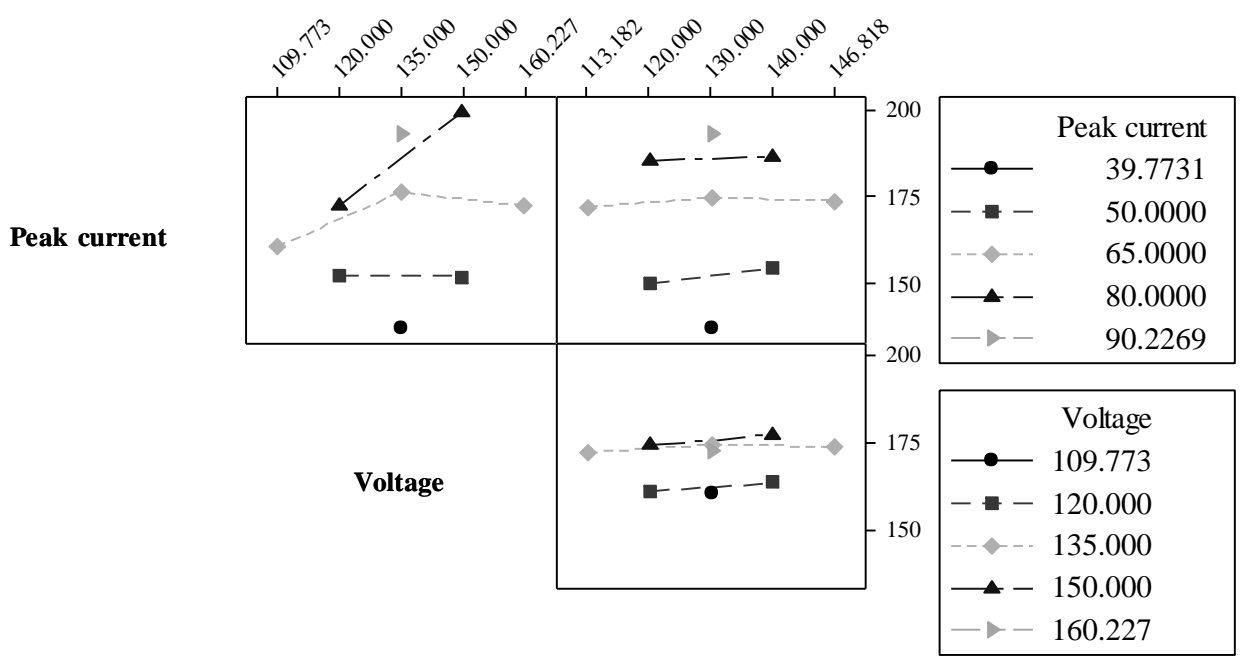

Frequency

Figure 4: TWR interaction plot for stainless steel using brass electrode.

Figure 5 shows the MRR of micro holes obtained on stainless steel using tungsten carbide and brass electrodes as a function of the Process Index. The trend is parabolic and the correlation index $\left(\mathrm{R}^{2}\right)$ is satisfactory. The points are distributed in two regions: micro holes obtained using tungsten carbide electrode are in the bottom region of the graph while holes 
obtained using brass electrode are in the top region. The different distribution is due to both different process parameters used and different thermal and electrical characteristics of the electrodes. Using brass electrodes the Process Index is higher, leading to an higher MRR than tungsten carbide one.

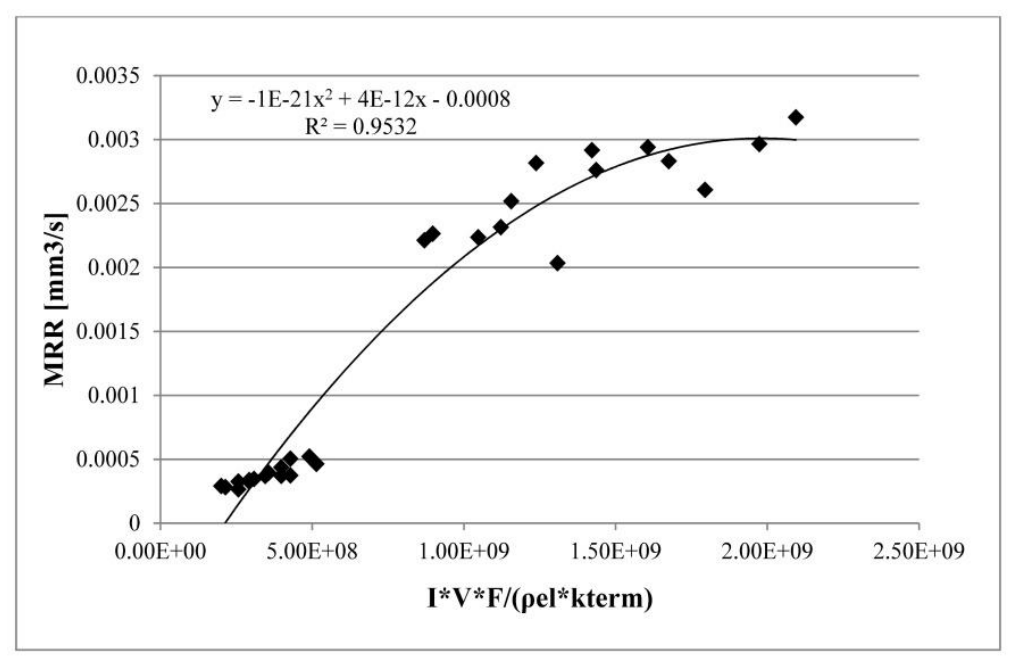

Figure 5: MRR of micro holes on stainless steel as a function of the Process Index.

Regarding TWR (figure 6), the trend is similar to MRR with a very high correlation index. As a general remark, brass electrodes permit a faster micro drilling process despite of the electrode wear. 


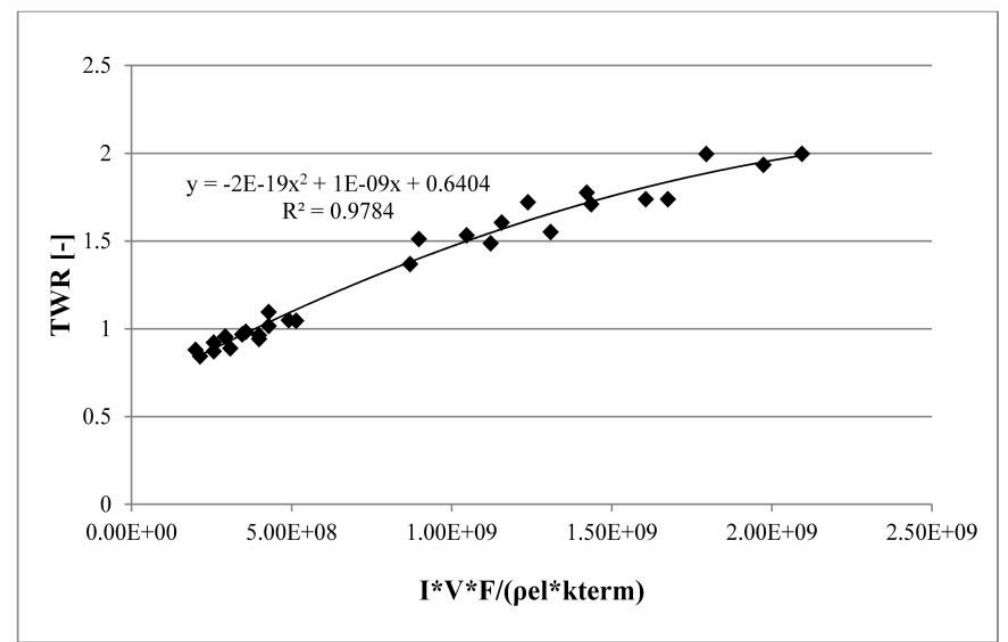

Figure 6: TWR of micro holes on stainless steel as a function of the Process Index.

Finally, the geometrical characteristics of the holes in terms of DOC and TR are reported together in figure 7. The curves confirm a parabolic trend: increasing the Process Index the accuracy of the top and bottom diameters becomes inferior. Diametral overcut and taper rate of the holes obtained using brass electrode are larger than tungsten carbide ones.

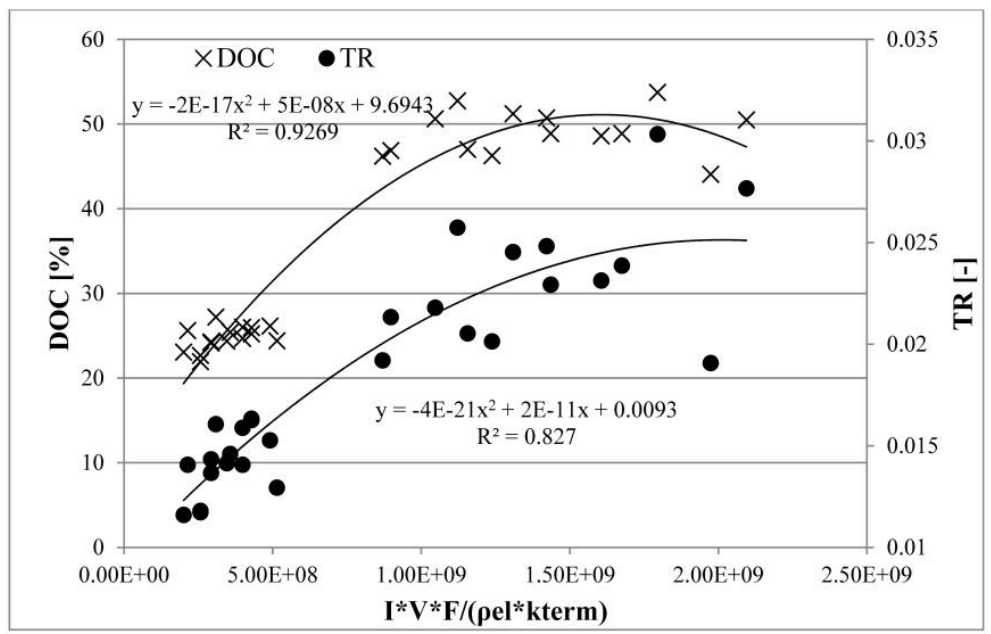

Figure 7: DOC and TR of micro holes on stainless steel as a function of the Process Index.

\subsection{Process performance evaluation for tungsten carbide workpiece}


Figures 8-11 show the interaction plots for MRR and TWR obtained on tungsten carbide workpieces using tungsten carbide and brass electrodes respectively.

ANOVA confirmed the influence of the process parameters on MRR and TWR for both the electrode types. Also in this case, a large data scatter in machining time was observed when brass electrode is used.

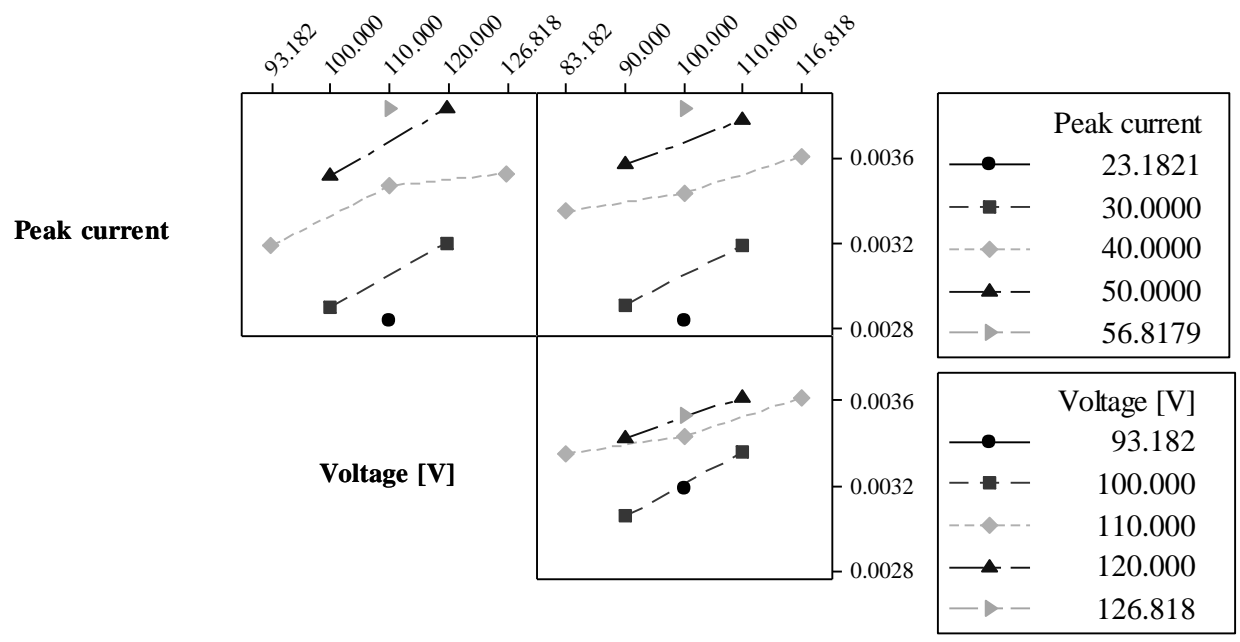

Frequency $[\mathbf{k H z}]$

Figure 8: MRR interaction plots for tungsten carbide using tungsten carbide electrode.

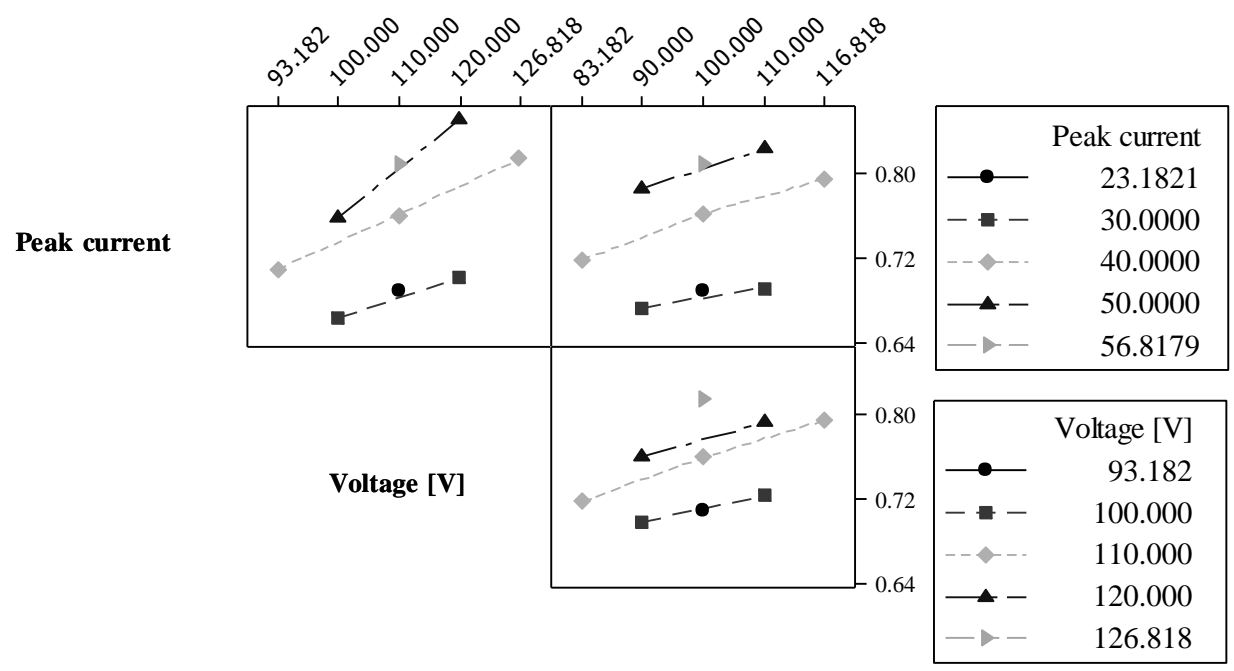

Frequency $[\mathrm{kHz}]$ 
Figure 9: TWR interaction plots for tungsten carbide using tungsten carbide electrode.

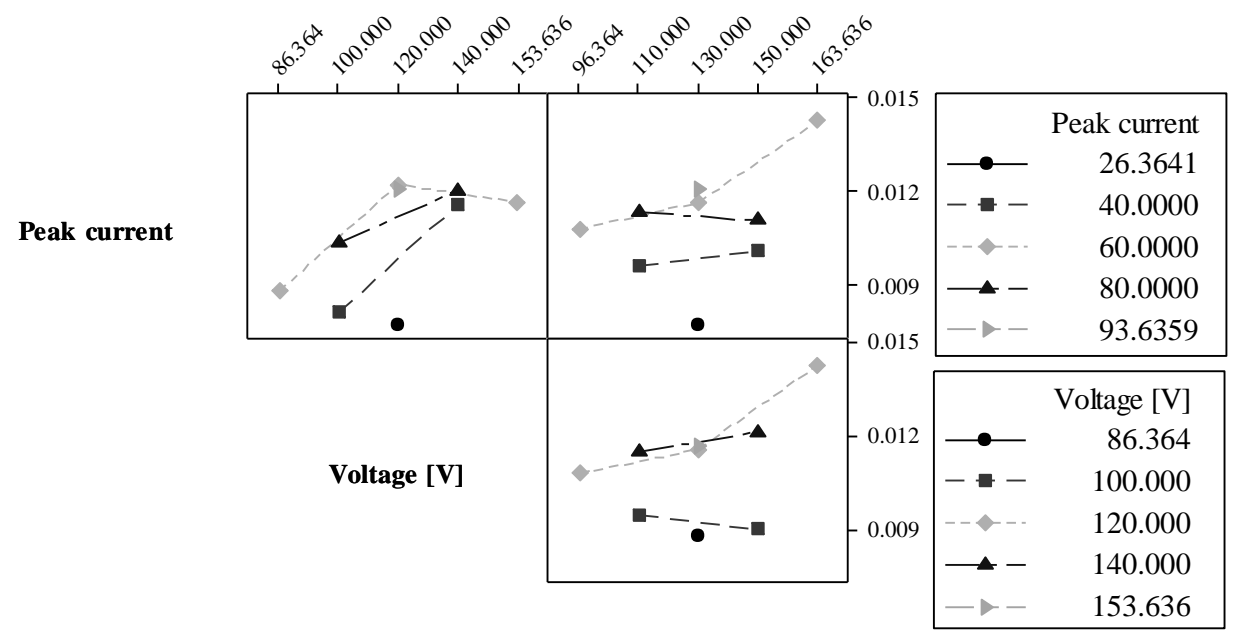

Frequency $[\mathbf{k H z}]$

Figure 10: MRR interaction plots for tungsten carbide using brass electrode.

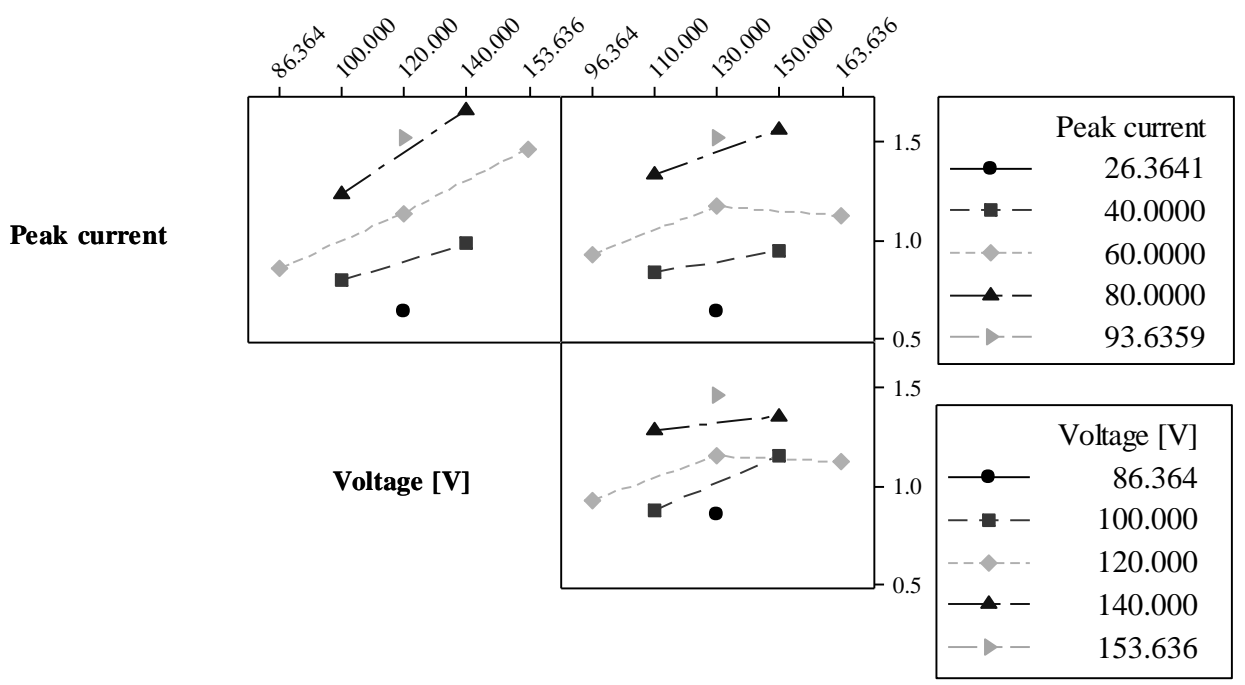

Frequency $[\mathbf{k H z}]$

Figure 11: TWR interaction plots for tungsten carbide using brass electrode.

Figures 12 and 13 show respectively MRR and TWR of micro holes on tungsten carbide as a function of the Process Index. A parabolic trend of MRR as a function of the Process Index is confirmed also in this case. As a general consideration, increasing the Process Index, the 
drilling process becomes faster. At the same time, an increase in the Process Index causes a worsening of the electrode wear.

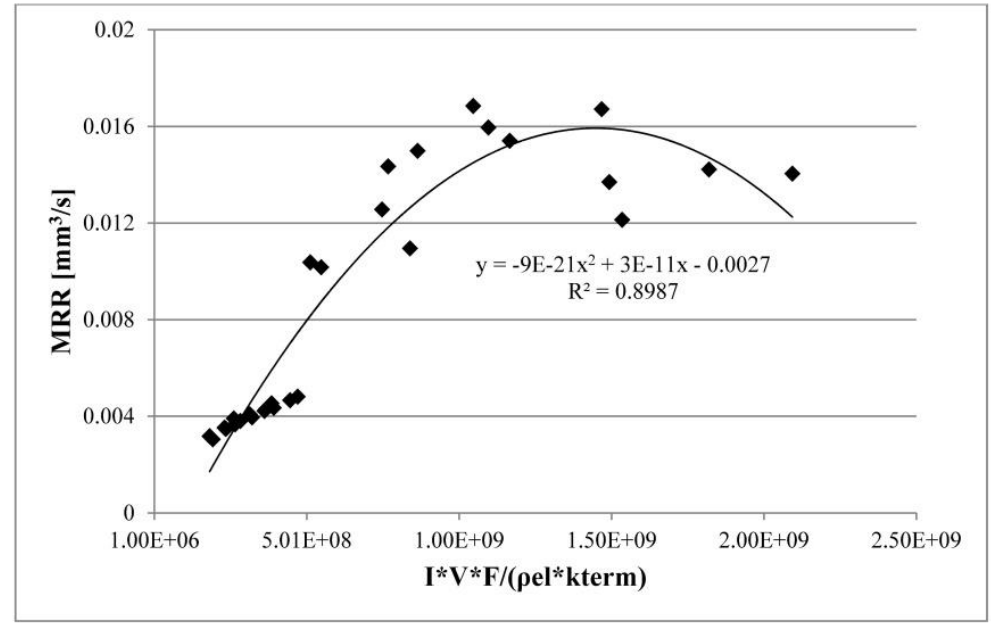

Figure 12: MRR of micro holes on tungsten carbide as a function of the Process Index.

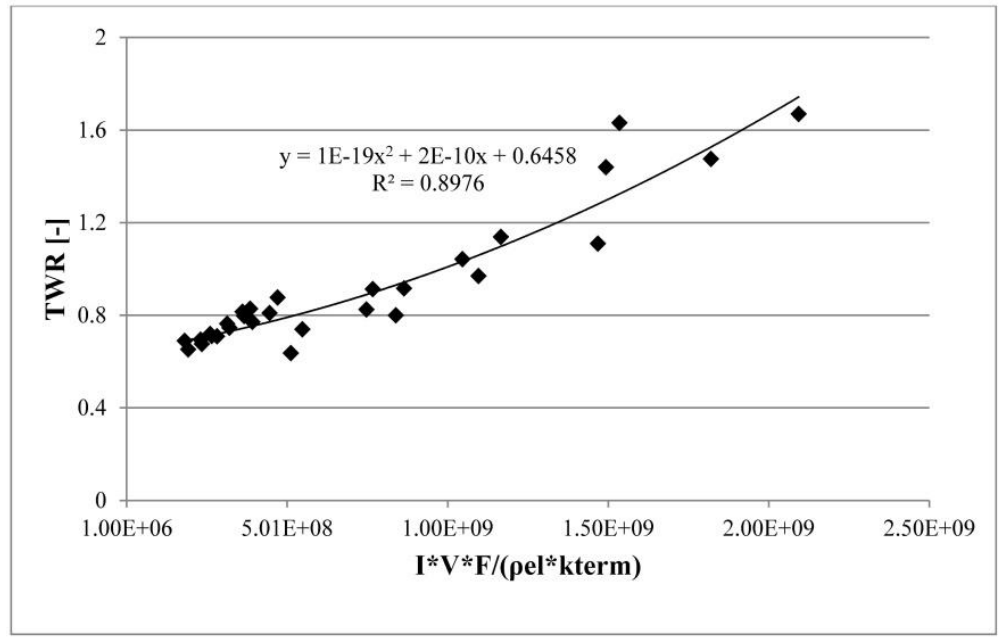

Figure 13: TWR of micro holes on tungsten carbide as a function of the Process Index.

Figure 14 shows DOC and TR of micro holes on tungsten carbide as a function of the Process Index. A large data scatter was observed. Finally, the accuracy of the micro holes get worse increasing the Process Index. 


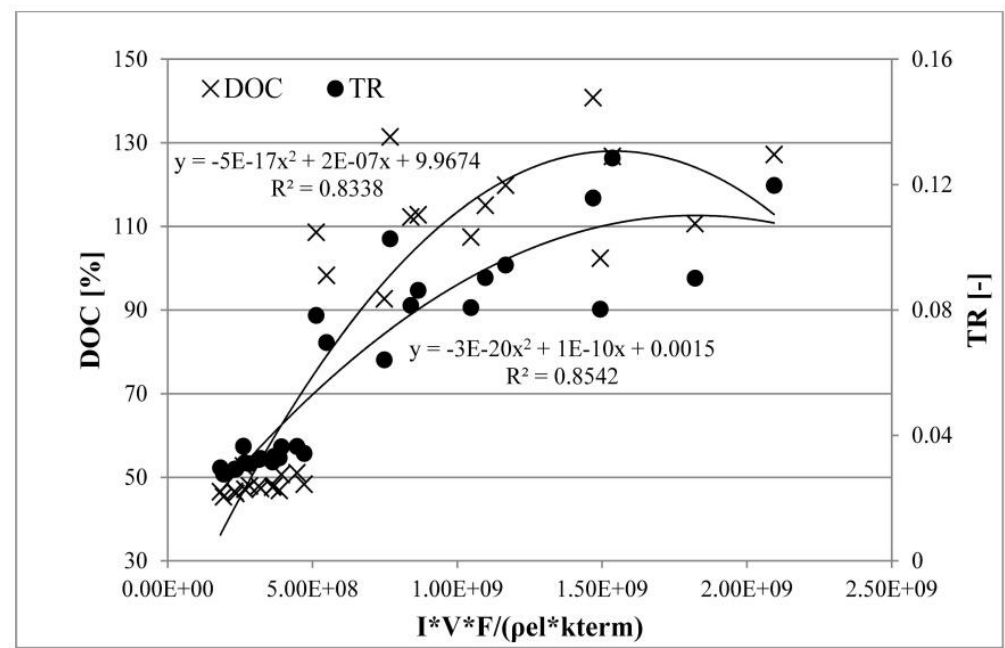

Figure 14: DOC and TR of micro holes on tungsten carbide as a function of the Process Index.

\subsection{Comparison between stainless steel and tungsten carbide workpieces}

In this section, a comparison of the process performance between stainless steel and tungsten carbide workpieces using the two types of electrode is discussed.

Stainless steel micro drilling process occurs in a more difficult way with respect to tungsten carbide workpiece, but leading to a positive effect on TWR (figure 15).

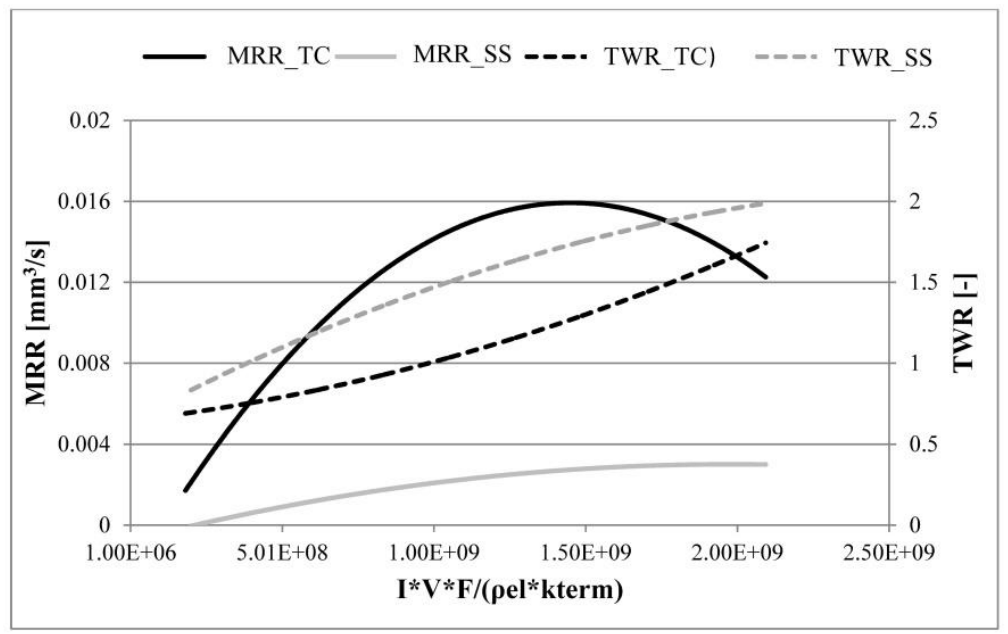


Figure 15: MRR and TWR of micro holes on stainless steel and tungsten carbide as a function of the Process Index.

Concerning the geometrical characteristics, figure 16 shows DOC and TR of micro holes on stainless steel and tungsten carbide as a function of the Process Index. As a general remark, the micro holes are more accurate in the conditions in which the removal of material is more difficult.

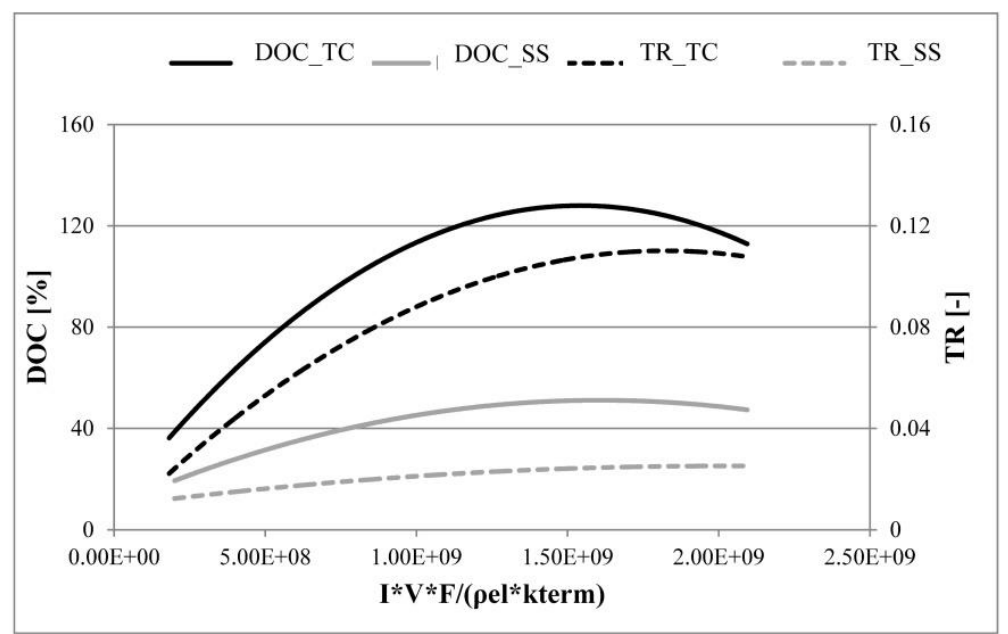

Figure 16: DOC and TR of micro holes on stainless steel and tungsten carbide as a function of the Process Index.

The effects of the physical properties of electrode and workpiece materials on process and geometric performances are summarized in table 5. The table is divided in two sections: the first one (top) takes into account the physical properties of the electrode materials, the second one (bottom) the properties of the workpiece.

Table 5: Effects of physical properties of electrode and workpiece materials on process and geometric performances. 


\section{MRR TWR DOC TR}

\section{个 $k_{\text {term }} \uparrow \hat{\uparrow} \uparrow \hat{\uparrow}$}

Electrode
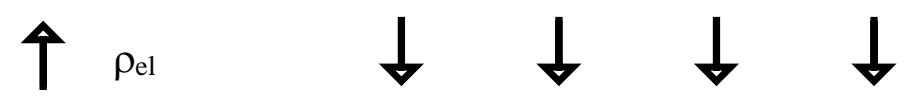

Material
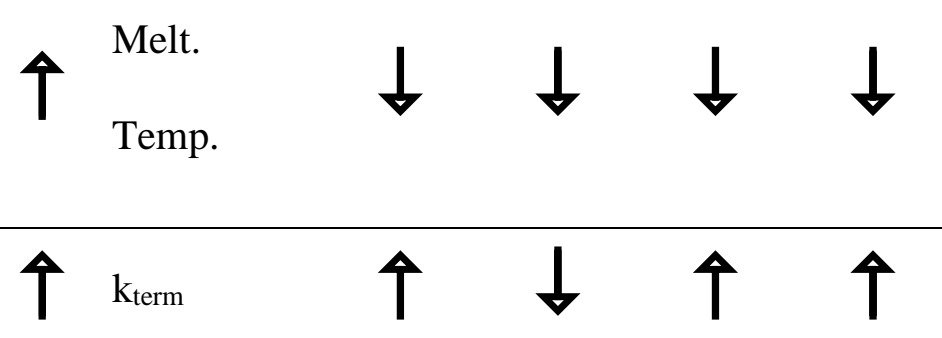

Workpiece $\quad \uparrow \rho_{\mathrm{el}} \downarrow \downarrow \downarrow \downarrow \downarrow$

Material

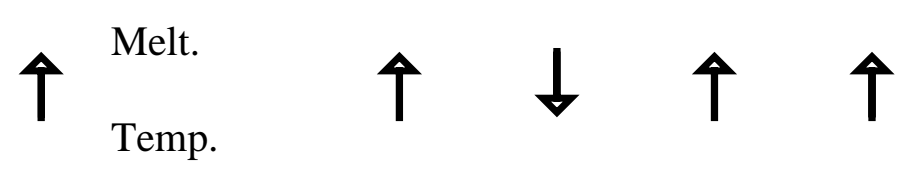

The effects of physical properties on the performances show a fully matching only considering the electrical resistivity. In other words, when the electrical resistivity rises (both for workpiece and electrode material) all the performance indexes decreases. Referring to the other physical properties, different effects on the performances can be observed when workpiece or electrode materials are considered.

\section{Conclusive remarks}

A study of the process performance of micro-EDM drilling on both stainless steel and tungsten carbide was carried out. The influence of electrode material and process parameters on material removal rate, tool wear ratio, diametral overcut and taper rate was investigated. 
An overall Process Index based on both process parameters and electrode material properties was defined. This index fits with a good correlation level all the process performance and geometric indicators for both the workpiece materials.

MRR and TWR increase for increasing values of the Process Index for both the workpiece materials. The brass electrode always allows a higher MRR with respect to the tungsten carbide one but is affected by a more severe TWR; this is due to the higher electrical conductivity and to the use of higher discharge energy.

The same considerations can be drawn for the geometrical characteristics of micro holes: DOC and TR are larger increasing the Process Index for both stainless steel and tungsten carbide workpieces.

Basing on the results previously described, it is possible to state that electrical resistivity, thermal conductivity and melting point of both electrode and workpiece materials have a significant effect on the performance.

\section{References}

Chiang, H.L., Wang, J.J.J., 2011. An analysis of overcut variation and coupling effects of dimensional variable in EDM process. Int J Adv Manuf Technol 55, 935-943.

Denkena, B., Hoffmeister, H.W., Reichstein, M., Illenseer, S., Hlavac, M., 2006. Micromachining processes for microsystem technology. Microsyst Technol. 12, 659-664.

D’Urso, G., Longo, M., Maccarini, G., Ravasio, C., 2011. Electrical discharge machining of micro holes on titanium sheets. In Proceedings of the 5th International Conference on Microand Nanosystems, ICONE14, Washington, DC, USA.

D’Urso, G., Ravasio, C., Maccarini, G., 2014. Process performance of micro-EDM drilling of stainless steel. Int J Adv Manuf Technol. 72, 1287-1298. 
Engqvist, H., Ederyd, S., Axen, N., Hogmark, S., 1999. Grooving wear of single-crystal tungsten carbide. Wear 230, 165-174.

Hoffmeister, H.W., Illenseer, S., 2004. Burr formation and reduction in micro drilling of metals. Prod Eng WGP 11, 1-4.

Hwang, Y.L., Kuo, C.L., Hwang, S.F., 2010. Fabrication of a micro-pin array with high density and high hardness by combining mechanical peck-drilling and reverse-EDM. Journal of Materials Processing Technology 210, 1103-1130.

Jahan, M.P., Rahman, M., Wong, Y.S., 2011. A review on the conventional and microelectro discharge machining of tungsten carbide. International Journal of Machine Tools \& Manufacture 51, 837-858.

Jahan, M.P., Wong, Y.S., Rahman, M., 2009. A study on the quality micro-hole machining of tungsten carbide by micro-EDM process using transistor and RC-type pulse generator. Journal of Materials Processing Technology 209, 1706-1716.

Jahan, M.P., Wong, Y.S., Rahman, M., 2009. A study on the fine-finish die-sinking microEDM of tungsten carbide using different electrode materials. Journal of Materials Processing Technology 209, 3956-3967.

Kuppan, P., Rajadurai, A., Narayanan, S., 2008. Influence of EDM process parameters in deep hole drilling of Inconel 718. International Journal Advanced Manufacturing Technology 38, 74-84.

Lee, S.H., Li, X., 2003. Study of the surface integrity of the machined workpiece in the EDM of tungsten carbide. Journal of Materials Processing Technology 139, 315-321. Liu, K., Li, X.P., 2011. Ductile cutting of tungsten carbide. Journal of Materials Processing Technology 113, 348-354. 
Liu, K., Li, X.P, Rahman, M., Liu, X.D., 2004. A study of the cutting modes in the grooving of tungsten carbide. International Journal of Advanced Manufacturing and Technology 24, $321-326$.

Mahardika, M., Tsujimoto, T., Mitsui, K., 2008. A new approach on the determination of ease of machining by EDM processes. Int. J. of Machine Tools \& Manufacture 48, 746-760. Mahdavinejad, R.A., Mahdavinejad, A., 2005. ED machining of WC-Co. Journal of Materials Processing Technology 162-163, 637-643.

Pradhan, B.B., Masanta, M., Sarkar, B.R., Bhattacharyya, B., 2009. Investigation of electrodischarge micro-machining of titanium super alloy. International Journal Advanced Manufacturing Technology 41, 1094-1106.

Schuberta, A., Zeidlera, H., Hahna, M., Hackert-Oschätzchena, M., Schneiderb, J., 2013. Micro-EDM Milling of Electrically Nonconducting Zirconia Ceramics. Procedia CIRP 6, 297-302.

Sona, S.M., Limb, H.S., Kumarb, A.S., Rahmanb, M., 2007. Influences of pulsed power condition on the machining properties in micro EDM. Journal of Materials Processing Technology 190, 73-76.

Tong, H., Li, Y. et al, 2013. Mechanism design and process control of micro EDM for drilling spray holes of diesel injector nozzles. Precision Engineering 37, 213-221. Trych, A, 2013. Further Study of Carbon Fibres Electrodes in Micro Electrical Discharge Machining. Procedia CIRP 6, 309-313.

Weule, H., Fleischer, J., Bookhholz, C., Knoll, M., Elsner, J., Tritschler, H. et al, 2004. International state of the art of micro production technology. Prod Eng Res Dev XI, 29-36. Yilmaz, O.M., Okka, A., 2010. Effect of single and multi-channel electrodes application on EDM fast hole drilling performance. International Journal Advanced Manufacturing Technology 51, 185-194. 
Yonga, T.H.L., Longa, Z., Baoquanb, L., 2013. Mechanism design and process control of micro EDM for drilling spray holes of diesel injector nozzles. Precision Engineering 37, 213- 221. 


\section{Figure Captions}

Figure 1: MRR interaction plots for stainless steel using tungsten carbide electrode.

Figure 2: TWR interaction plots for stainless steel using tungsten carbide electrode.

Figure 3: MRR interaction plots for stainless steel using brass electrode.

Figure 4: TWR interaction plot for stainless steel using brass electrode.

Figure 5: MRR of micro holes on stainless steel as a function of the Process Index.

Figure 6: TWR of micro holes on stainless steel as a function of the Process Index.

Figure 7: DOC and TR of micro holes on stainless steel as a function of the Process Index.

Figure 8: MRR interaction plots for tungsten carbide using tungsten carbide electrode.

Figure 9: TWR interaction plots for tungsten carbide using tungsten carbide electrode.

Figure 10: MRR interaction plots for tungsten carbide using brass electrode.

Figure 11: TWR interaction plots for tungsten carbide using brass electrode.

Figure 12: MRR of micro holes on tungsten carbide as a function of the Process Index.

Figure 13: TWR of micro holes on tungsten carbide as a function of the Process Index.

Figure 14: DOC and TR of micro holes on tungsten carbide as a function of the Process Index.

Figure 15: MRR and TWR of micro holes on stainless steel and tungsten carbide as a function of the Process Index.

Figure 16: DOC and TR of micro holes on stainless steel and tungsten carbide as a function of the Process Index. 


\section{Table Captions}

Table 1: Physical properties of electrodes' material.

Table 2: Fixed process parameters.

Table 3: Combination of Technologies based on DOE for AISI304 workpiece.

Table 4: Combination of technologies based on DOE for tungsten carbide workpiece.

Table 5: Effects of physical properties of electrode and workpiece materials on process and geometric performances. 